\title{
Selection and justification of priority tasks of biogas plant management taking into account technological risks
}

\author{
Valery Lubentsov*, Ekaterina Ozhogova, Elena Lubentsova, and Evgeniy Shakhray \\ Kuban State Technological University, 2 Moskovskaya Street, Krasnodar, 350972, Russia
}

\begin{abstract}
This article is devoted to the selection and justification of priority applied control tasks in the automation of a biogas plant (BP), taking into account the risks of loss of control. The types of technological risks in the management of fermentation processes in methane tanks are considered. A reasoned analysis of quantitative risk assessments using the pairwise comparisons method is carried out. The use of the method of consistency of expert opinions in the algorithm for solving the problem of selection and justification made it possible to conduct a strict analysis of the consistency of expert opinions and to identify whether the obtained estimates are random or not. Using the obtained information models for the regime parameters of anaerobic digestion processes, the relevance of developing a better control system for the optimal temperature regime of substrate heating, temperature stabilization in the methane tank, and the rational end time of the fermentation process is justified. The results obtained are in the best agreement with the improvement of the BP process management efficiency.
\end{abstract}

\section{Introduction}

The development of modern society is provided, first of all, by the energy base. The threat of an energy crisis on a global scale makes the problem of developing and popularizing renewable energy sources urgent. Even today, in many countries, the active use of such sources is one of the main priorities of energy policy. Inefficient waste management and growing problems with environmental pollution are the result of the irrational use of natural resources around the world. If by 2010 the contribution of biomass to the total energy consumption in the world was up to $12 \%$, the forecast for the growth of biomass as a source of renewable energy in the world suggests reaching $23.8 \%$ by 2040 [1]. Biogas plants are one of the most important means of developing bioenergy. Currently, various biogas plants schemes have been developed and successfully applied in the world.

Biogas plants (BP) are of particular interest for livestock enterprises. Almost all the waste of these enterprises, especially the waste of large farms, is of organic origin, and can be disposed of by anaerobic digestion. Thanks to the use of biogas technologies, it is possible to achieve the utilization of organic waste with the production of biogas and high-

\footnotetext{
* Corresponding author: vf.lubentsov@yandex.ru
} 
quality fertilizer, which has a less aggressive effect on plants than before processing, helps to reduce the emission of unpleasant odors and improve the environmental situation.

By now, many technological BP schemes have been developed. For farmers who want to use $\mathrm{BP}$, there is a need to make a deep analysis of all the technologies presented, to determine the most cost-effective and suitable solution for the climatic conditions of this region. This fact is significantly complicated by the lack of ready-made solutions for the automation of these plants, which hinders the implementation of biogas technologies, taking into account the peculiarities of the region. The presence of reasonable tasks solved in automation and automatic control systems (ACS) for fermentation processes contributes to ensuring the modern technical level, quality and efficiency of BP during their operation.

For a long time, agriculture has not been an attractive area for the application of information technology (IT) in automation due to the long production cycle, subject to natural risks and large crop losses during cultivation, the inability to use high-quality information and a small amount of quantitative information for operational decisionmaking. The use of IT in the agro-industrial complex was limited to the use of computers and software, mainly for financial management and tracking commercial transactions [2, 3]. Not so long ago, farmers began to use digital technologies to monitor agricultural crops and support decision - making of applied problems of agricultural production, for example, to determine the rational area of the cultivated land plot [4]. BP automation is becoming a new class of tasks to be solved and its practical focus on the development of alternative energy sources. As a result, it is relevant to choose, justify priority tasks and make decisions on automation and management of BP based on advanced IT.

The scientific novelty of the used problem solving technology is as follows:

- in the justification of a new class of tasks to be solved in the context of a constant increase in prices for basic energy carriers and the depletion of the Earth's hydrocarbon resources in the presence of cheap raw materials and the accumulation of waste in the agroindustrial complex;

- the basis of the technology used does not require the involvement of a large number of experts;

- in ensuring an increase in the stability of the result of solving the problem by applying the method of consistency of expert judgments developed by us in the solution algorithm [5].

\section{Justification of the chosen method of solving the problem}

The suggested method of selecting the priority tasks of automatic control and regulation of the BP is based on a method that makes it possible to take into account many factors. Since there are several different methods of quantitative and expert evaluation of the choice, there is uncertainty in the choice of the method of solving the problem and, therefore, there is a risk of making an irrational decision when choosing problems. To reduce the risk, it is necessary to eliminate the uncertainty in the choice of the solution method, it means to remove the inconsistency between the results of solutions by different methods and thus ensure the stability of the result obtained. To increase the stability of the solution result, it is suggested to use the method of pairwise comparisons, which implements a method of processing information by increasing the consistency of expert opinions and conducting their hierarchical evaluation, which allows you to get the priority of tasks for their solution.

In an article by specialists of the German company Sartorius BBI Systems GmbH [6] on risk analysis and management, it is noted that risk management together with the fermentation process management system allows to increase the efficiency of management decisions. At the same time, the analysis of existing developments shows that almost at the stage of automated control systems (ACS) design, when choosing and justifying the tasks 
of regulation and management, possible risks from the loss of control of the BP are not considered. In this connection, it is not possible to identify the impact of each of the decisions on the overall effectiveness due to their aggregate influence. To increase the validity of the choice of the most significant tasks of managing the interconnected BP processes in the designed automated control systems, we will use a pairwise comparison of the risks from loss of control.

\section{Comparative analysis of quantitative risk assessments in the management of a biogas plant}

The process of anaerobic digestion is a complex technological process. For the normal course of fermentation, it is necessary to maintain optimal conditions in the methane tank, which are provided by the functioning of control systems at different interrelated stages of biogas technology. If the modes deviate from the optimal ones, risks are possible at each of the stages. The main ones are the following:

- risks associated with choosing a non-optimal anaerobic digestion regime $-\mathrm{R}_{11}$;

- risks associated with the choice of a non-optimal mode of substrate supply to methane $\operatorname{tanks}-\mathrm{R}_{12}$;

- risks associated with the violation of the heating system of the methane tank and its insulation $-\mathrm{R}_{13}$;

- risks associated with disruption of the biogas collection system- $\mathrm{R}_{14}$;

- risks associated with violations of the biogas discharge technology, the cleaning system and the operation of other auxiliary equipment $-\mathrm{R}_{15}$.

A reasonable choice of a rational decision to manage the process is associated with the assessment of the most significant risk from the above groups. The comparison of risk assessments was carried out by pair-wise comparison using the analytic hierarchy process (AHP) [7]. For a pair-wise comparison of indicators, we use a scale that contains numerical indicators from 1 to 9 and their inverse values [7]. Expert judgments are expressed in the integers of this scale. A positive aspect of the AHP is the ability to check expert assessments for consistency, which may appear when the expert fills in the comparison matrix (CM). Taking into account the rating scale, the comparison of the impact of the main characteristics on efficiency was carried out according to the following principles [8]:

1 - the considered risk characteristic affects the effectiveness to the same extent;

3 - the considered risk characteristic slightly reduces the effectiveness compared to the other;

5 - the considered risk characteristic noticeably reduces the effectiveness compared to the other;

7 - the considered risk characteristic significantly reduces the effectiveness compared to the other;

9 - the considered risk characteristic greatly reduces the effectiveness compared to the other;

2, 4, 6, 8 - the corresponding intermediate values.

To establish the consistency of expert estimates, the deviation of the maximum eigenvalue $\lambda_{\max } \mathrm{CM} \mathrm{M}\left(a_{i j}\right)$ from the order $\mathrm{n}$ of this matrix is calculated. The uniformity of judgments is expressed by the consistency index (CI) and the consistency ratio (CR), calculated by the formulas:

$$
\mathrm{CI}=\left(\lambda_{\max }-n\right) /(n-1) ; \mathrm{CR}=\mathrm{CI} / \mathrm{E}(\mathrm{CM}),
$$

where $\lambda_{\max }$ - eigenvalue of the matrix; 
$n$ - number of items to compare;

$\mathrm{E}(\mathrm{CM})$ - expected value CI CM.

Expert opinions are considered to be consistent with the value of $\mathrm{CR} \leq 10 \%$. The consistency of the matrices of pair-wise comparisons is achieved by adjusting their eigenvalues [5]. The positive aspect of this adjustment method is that there is no need to revise all the values of the matrix to improve its consistency. As a result of the expert survey, a matrix of risk comparisons was obtained and the results for the group of risks associated with violations in the systems for ensuring the optimal mode of the fermentation process, shown in Table 1.

Table 1. Comparison matrix of risks of a group of risks associated with violations in the systems for ensuring the optimal mode of the fermentation process.

\begin{tabular}{|c|c|c|c|c|c|c|c|}
\hline $\begin{array}{c}\text { Compared } \\
\text { options }\end{array}$ & $\begin{array}{r}\text { Risk } \\
\mathrm{R}_{11}\end{array}$ & $\begin{array}{c}\text { Risk } \\
\mathrm{R}_{12}\end{array}$ & $\begin{array}{r}\text { Risk } \\
\mathrm{R}_{13}\end{array}$ & $\begin{array}{r}\text { Risk } \\
\mathrm{R}_{14}\end{array}$ & $\begin{array}{r}\text { Risk } \\
\mathrm{R}_{15}\end{array}$ & $\begin{array}{l}\text { Priority } \\
\text { vector } \\
\text { estimates }\end{array}$ & $\begin{array}{l}\text { Normalized } \\
\text { priority } \\
\text { vector } \\
\text { estimates }\end{array}$ \\
\hline Risk $\mathrm{R}_{11}$ & 1 & 2 & 4 & 8 & 9 & 3.565205 & 0.477798 \\
\hline Risk $\mathrm{R}_{12}$ & $1 / 2$ & 1 & 3 & 5 & 7 & 2.208167 & 0.295932 \\
\hline Risk $\mathrm{R}_{13}$ & $1 / 4$ & $1 / 3$ & 1 & 2 & 4 & 0.922108 & 0.123578 \\
\hline Risk R 14 & $1 / 8$ & $1 / 5$ & $1 / 2$ & 1 & 2 & 0.478176 & 0.064084 \\
\hline Risk $\mathrm{R}_{15}$ & $1 / 9$ & $1 / 7$ & $1 / 4$ & $1 / 2$ & 1 & 0.288081 & 0.038608 \\
\hline Sum & 1.9861 & 3.6762 & 8.7500 & 16.5000 & 23,0000 & 7.425790 & 1.000000 \\
\hline
\end{tabular}

The information model (IM) obtained on the basis of a pair-wise comparison of risks has the form:

$$
\mathrm{IM} 1=0.477798 \cdot \mathrm{R}_{11}+0.295932 \cdot \mathrm{R}_{12}+0.123578 \cdot \mathrm{R}_{13}+0.064084 \cdot \mathrm{R}_{14}+0.038608 \cdot \mathrm{R}_{15} .
$$

The option with the maximum value of the priority vector is considered a priority. It can be seen from (1) that the first variant really surpasses the other analogues in terms of basic characteristics.

The resulting weight coefficients show the significance of the risks $R_{11}$ and $R_{12}$, since their total weight is $77.4 \%(47.8 \%+29.6 \%)$. The consequences of risks $\mathrm{R}_{13}, \mathrm{R}_{14}, \mathrm{R}_{15}$ are considerably less significant than the risks $R_{11}$ and $R_{12}$, which is explained by the conduct of these operations after the start or end of the process during the operation of support equipment. The results obtained are in the best agreement with the essence of the applied problems of industrial BP management and the requirements for their solution. The suboptimality of the fermentation modes (risk $\mathrm{R}_{11}$ ) is determined by the violation of the factors affecting gas production. These factors, from a technical point of view, most often include the supply systems, heating of the substrate and mixing, temperature and $\mathrm{pH}$, and the composition of the substrate mixture. The supply of the substrate (s) to the apparatus (risk $\mathrm{R}_{12}$ ) serves as an effective control effect on the fermentation process. The control of the substrate supply should be carried out slowly and exclude both the supply of large portions of cold substrate and changes in the composition of the substrate. The factors affecting the production of gas are most often the substrate supply systems (too much loading of the fermenter (over-feeding), or too little loading of the substrate (underfeeding)), heating of the substrate, temperature and mixing of the medium in the apparatus. Management of fermentation in many cases is reduced to maintaining the concentration of the substrate within the $s_{\min } \leq s \leq s_{\max }$ by introducing certain portions as the composition of the substrate mixture changes during fermentation. 
Let us proceed to the analysis of the consequences of risks directly related to the fermentation processes in methane tanks. The risks associated with the loss of control of the fermentation process, as a rule, reduce the yield of gas and fertilizer, and, consequently, reduce the economic efficiency of the BP. To control and regulate the fermentation process, the following risks are identified as the main ones: risks associated with poor control of the heating temperature of the supplied substrate in the fermenter $-R_{21}$; risks associated with the loss of accuracy of controlling the temperature of the medium in the fermenter $-R_{22}$; risks associated with the loss of control of the fermentation time $-\mathrm{R}_{23}$; risks associated with the lack of control of changes in the composition of the substrate in the fermenter $\mathrm{R}_{24}$; risks associated with insufficient speed of obtaining information about the concentration of sulfur and ammonia when changing the composition of the substrate mixture before the start of the fermentation process and the formation of fatty acids or the concentration of $\mathrm{H}^{+}$ions in the produced gas, especially when changing the composition of the substrate mixture $-\mathrm{R}_{25}$.

Using expert information for these risks, a comparison matrix is obtained and the results are summarized in Table 2.

Table 2. A comparison matrix of the risks of the second group and the assessment of the priority vector.

\begin{tabular}{|c|c|c|c|c|c|c|c|}
\hline $\begin{array}{c}\text { Compared } \\
\text { options }\end{array}$ & $\begin{array}{r}\text { Risk } \\
\mathrm{R}_{11}\end{array}$ & $\begin{array}{c}\text { Risk } \\
\mathrm{R}_{12}\end{array}$ & $\begin{array}{r}\text { Risk } \\
\mathrm{R}_{13}\end{array}$ & $\begin{array}{r}\text { Risk } \\
\mathrm{R}_{14}\end{array}$ & $\begin{array}{r}\text { Risk } \\
\mathrm{R}_{15}\end{array}$ & $\begin{array}{l}\text { Priority } \\
\text { vector } \\
\text { estimates }\end{array}$ & $\begin{array}{l}\text { Normalized } \\
\text { priority } \\
\text { vector } \\
\text { estimates }\end{array}$ \\
\hline Risk $\mathrm{R}_{21}$ & 1 & 2 & 4 & 8 & 9 & 3.565205 & 0.481756 \\
\hline Risk $\mathrm{R}_{22}$ & $1 / 2$ & 1 & 2 & 4 & 8 & 2.000000 & 0.270254 \\
\hline Risk $R_{23}$ & $1 / 4$ & $1 / 2$ & 1 & 2 & 5 & 1.045640 & 0.141294 \\
\hline Risk $R_{24}$ & $1 / 8$ & $1 / 4$ & $1 / 2$ & 1 & 3 & 0.542236 & 0.073271 \\
\hline Risk $R_{25}$ & $1 / 9$ & $1 / 8$ & $1 / 5$ & $1 / 3$ & 1 & 0.247352 & 0.033425 \\
\hline Sum & 1.9861 & 3.8750 & 7.7000 & 15.3333 & 26.0000 & 7.400432 & 1.000000 \\
\hline
\end{tabular}

The information model obtained on the basis of a pair-wise comparison of the second group of risks has the form:

$$
\mathrm{IM} 2=0.481756 \cdot \mathrm{R}_{21}+0.270254 \cdot \mathrm{R}_{22}+0.141294 \cdot \mathrm{R}_{23}+0.073271 \cdot \mathrm{R}_{24}+0.033425 \cdot \mathrm{R}_{25} \text {. }
$$

The option with the maximum value of the priority vector is considered a priority. It can be seen from (2) that the first option really surpasses the other analogues in terms of basic characteristics.

As a result of the analysis of this group of risks, it was revealed that during the fermentation process, the most significant losses will be from poor-quality control of the heating temperature of the supplied substrate in the fermenter $\left(R_{21}\right)$; risks associated with the loss of accuracy of control of the medium temperature in the fermenter $\left(R_{22}\right)$; risks associated with the loss of control of the duration of the fermentation process - the time of the optimal end of the process $\left(\mathrm{R}_{23}\right)$. Their total weight is $89.3 \%(48.2 \%+27 \%+14.1$ $\%)$.

Confirmation of the received estimates is the following.

The temperature of the substrate in the fermenter must be regulated with high accuracy, since anaerobic microorganisms are very sensitive to its sharp fluctuations. This is due to the rate of adaptation of the biomass to new conditions. Maximum temperature fluctuations can lead to a slowdown in the process of anaerobic biochemical oxidation, and in critical conditions even to a complete stop in the formation of biogas, due to the death of anaerobic 
bacteria for the synthesis of methane [8]. Plants with a psychrophilic mode of operation due to the low temperature of $23{ }^{\circ} \mathrm{C}$ have a long fermentation period and a relatively small gas capacity. It is known [9] that the higher the temperature of the process, the more sensitive the bacteria are to its fluctuations. This follows from the relatively narrow maximum of the graph curve under the thermophilic regime.

Practice has shown that microorganisms are harmed, first of all, by a rapid change in temperature, and on the contrary, methanogenic microorganisms can, in the case of a slow change in temperature, adapt to its different values. Therefore, for the stability of the technological process, the absolute temperature is not so important, as the constancy of the temperature value is much more important. When choosing the temperature regime, it is necessary to provide reliable insulation suitable for the climatic conditions of the plant installation region, a system for automating the operation of the biogas plant and the heating system, in order to avoid sudden temperature fluctuations [10].

The temperature at which fermentation takes place has a significant effect on the time of the process. It is known the higher the temperature of the process is the faster the decomposition occurs. At the same time, the combined effect of the fermentation temperature and the fermentation time on the amount of gas produced should be noted. A short duration of fermentation under anaerobic conditions leads to incomplete processing of the substrate, an excessively long duration reduces the mass of waste disposed of and leads to economic losses [11]. Therefore, one of the main tasks for managing the BT regime is to determine the optimal duration of waste processing in the methane tank.

Losses associated with the control of the substrate supply should, if possible, exclude changes in the composition of the substrate. The latter is not an element of the automatic mode, since it concerns changing the diet of animals before it is fed to the fermenters. This is confirmed by the low risk value of $\mathrm{R}_{24}=7.3 \%$. The inability to automatically influence the concentration of sulfur and ammonia in the substrate mixture before starting the fermentation process and the formation of fatty acids in the produced gas is characterized by the lowest risk value of $\mathrm{R}_{25}=3.3 \%$.

\section{Conclusion}

The most important tasks of BT management, identified taking into account technological risks and the modular principle of the organization of biogas technology, are the control of the feed process and the set heating rate of the substrate fed to the BT reactors, stabilization or control of the optimal temperature regime in the reactor, as well as control of the mixing of the medium in the fermenter. Solving these problems requires ensuring a high degree of reliability and accuracy of functioning ACS. The effective functioning of such ACS, combined with the management of technological risks, will ensure the economical disposal of various types of organic waste. Less suitable tasks for solving in automatic mode are those related to ensuring the reliability of the heating system of the methane tank and its incomplete thermal insulation, as well as the system for collecting and unloading biogas, its purification and the operation of other auxiliary equipment (filters, gas tanks). These tasks are solved with the use of so-called closed strapping devices and the use of filters.

If the management of the BT does not eliminate the risks associated with the violation of the conditions for the preparation of raw materials, the heating system and the supply of the substrate, the temperature regime during the fermentation process and the operation of the gas tank, then the funds spent, literally, go to the sewer. When choosing BT management tasks, you should take into account the above-mentioned critical operations and strive to minimize the risks associated with them.

Since the priorities of the management tasks of the existing BT are not clear, the chosen method of paired comparisons in this case is applicable and allows you to determine the 
relative importance of different options and provides a basis for comparing each option with others, helping to rank the options. The numerical results obtained by this method are in the best agreement with the essence of the applied problems of BT management and the requirements for their solution.

\section{References}

1. GOST R 53790-2010. Non-traditional technologies. Biosolids energy. General technical requirements for biogas plants, Moscow, Russia: Standartinform (2019) URL: http://docs.cntd.ru/document/1200083240

2. Overview of digital technologies for the agro-industrial complex: from GIS to the Internet of things (2020) URL: http://integral-russia.ru/2019/10/28/tsifrovayaplatforma-razvitiya-agropromyshlennogo-kompleksa-kontseptsiya-i-osnovnye-tezisy

3. IT-technologies in agriculture URL: http://www.controlunion.ru/index.php/11novosti/425-it-tekhnologii-v-selskom-khozyajstve

4. E. Lubentsova, G. Masyutina, V. Lubentsov, A. Permyakov and M. Kozlova. IOP Conference Series: Earth and Environmental Science. Decision-making information support methodology for protective measures implementation in the AIC, 613, 012073 (2020)

5. E. Lubentsova, E. Ozhegova, V. Lubentsov, E. Shakhray and G. Masyutina. Modern high technologies. Method of matching paired comparison matrices based on components of their maximum eigenfrequency numbers, 7, 78-83 (2020)

6. L. Goren, K. Clapp. Next Generation Pharmaceutical. Risk and quality management a suppliers perspective, 114-115 (2005)

7. T. Saaty. Decision-making with dependencies and feedbacks: analytical networks, 360 (2009)

8. T. Saaty. Fuzzy sets and Systems. Eploring the interface between hierarchies, multiple objectives and fuzzy sets, 1, 57-68 (1978)

9. Systems for ensuring thermal conditions in anaerobic digestion reactors (2015) URL: https://nomitech.ru/articles-and-

blog/sistemy_obespecheniya_termicheskogo_rezhima_v_reaktorakh_anaerobnogo_sbr azhivaniya/

10. G. Osadchiy. Systems for ensuring the thermal regime in anaerobic digestion reactors (2015) URL: http://amureco.ru/ekoknigi/nauchno-populyarnye-stati/vozmozhnosti-pomodernizatsii-biogazovykh-ustanovok

11. Dmitry Borisovich Marchenko. Abstract of the dissertation for the degree of Candidate of Technical Sciences (05.20.01) - technologies and means of agricultural mechanization. Justification of technological and design parameters of equipment for the production of organic fertilizer and biogas from bird droppings, 25, Omsk, Russia (2009) 